\title{
Martina Wagner-EgelhaAF
}

\section{Ikonoklasmus}

\section{Autorschaft und Bilderstreit}

In Heiner Müllers Dramolett Die Hamletmaschine aus dem Jahr 1977 tritt Hamlet als „Hamletdarsteller“ ${ }^{1}$ auf. Er stellt sich gleichsam neben seine Rolle und zertrümmert ihr ganzes dramatisch-ästhetisches Bezugssystem: Ich bin nicht Hamlet. Ich spiele keine Rolle mehr. Meine Worte haben mir nichts mehr zu sagen. Meine Gedanken saugen den Bildern das Blut aus. Mein Drama findet nicht mehr statt. ${ }^{2}$ Die Hamletfigur, häufig als Urbild des reflektierenden Intellektuellen interpretiert, repräsentiert bei Müller den desillusionierten Künstler in der sozialistischen Gesellschaft, der keine Möglichkeiten mehr für sich und seine Arbeit sieht. ${ }^{3}$ Im 4. Bild des nur neun Seiten und fünf Bilder umfassenden, episodischen Stücks stößt man unvermittelt auf zwei irritierende Regieanweisungen. Der Hamletdarsteller sagt:

[...]

In der Einsamkeit der Flughäfen

Atme ich auf Ich bin

Ein Privilegierter Mein Ekel

Ist ein Privileg

Beschirmt mit Mauer

Stacheldraht Gefängnis

Fotografie des Autors.

Ich will nicht mehr essen trinken atmen eine Frau lieben einen Mann ein Kind ein Tier. Ich will nicht mehr sterben. Ich will nicht mehr töten.

Zerreißung der Fotografie des Autors ${ }^{4}$

\footnotetext{
1 Heiner Müller, „Die Hamletmaschine“, in Ders., Mauser, Berlin 1978, 89-97, hier 93.

2 Ebd.

3 Vgl. ebd. 97.

4 Ebd. 96.
} 
Welchem Autor dieser ikonoklastische Akt gilt, verrät der Text nicht. Geht es um die Instanz des Autors generell oder um einen bestimmten Autor? ${ }^{5}$ Da es von William Shakespeare keine Fotografien gibt, ist dann vielleicht die Fotografie des Autors Heiner Müller gemeint? Dieser Gedanke legt sich zumindest deshalb nahe, weil die in der zitierten Passage dargestellte Situation sehr genau derjenigen Müllers zum Zeitpunkt der Entstehung des Stücks entspricht. Aber doch steht in diesem Fall der individuelle Autor auch für die Instanz des Autors. Die politisch-kritische Lektüre, die in der Zerstörung des Autorbildes die Unmöglichkeit von Autorschaft in der Diktatur liest, verbindet sich in Müllers Hamletmaschine mit einer postdramatischen Verabschiedung der überkommenen ästhetischen Kategorien von Schauspiel, Rolle, Handlung und eben auch von Autorschaft. ${ }^{6}$ Die im Stück geübte politische Kritik weist einen generellen, nicht nur die Situation in der DDR betreffenden zeitdiagnostischen Index auf, insofern als die Position des Subjekts und mithin auch das Konzept von Autorschaft in der Postmoderne eine grundsätzliche Infragestellung erfahren haben.

Der 1943 geborene Schriftsteller Wilhelm Genazino kommt in seinem Essay Das Bild des Autors ist der Roman des Lesers aus dem Jahr 1994 auf die Zerreißung des Autorbildes in Müllers Hamletmaschine zurück. Und zwar bezieht er sich auf eine nicht näher bezeichnete Inszenierung des Stücks, in der sich der Hamletdarsteller - in einem auf der Bühne aufgestellten Fernsehapparat - ein Foto des Autors Heiner Müller vor das Gesicht hält und [...] dieses Foto anschließend selber zerstört. ${ }^{7}$ Für Genazino zeigt sich in dieser Szene ein grundsätzliches Problem: Die Zerreißung der Fotografie des Autors ist, so argumentiert er, ein hochtheatralischer Akt, der noch in der Negation des Bildes die Selbstdarstellung des Autors ermögliche. Genazino zieht hier eine Verbindungslinie zu Michel Foucaults für die neuere Autorschaftsdebatte zentralem Essay Was ist ein Autor?, in dem Foucault den Gedanken formuliert, dass das Werk, das einmal die Aufgabe hatte, unsterblich zu machen - hier wird auf das Modell vom Autor als Genie und quasi göttlichem Schöpfer angespielt - heute, also im Jahr 1969, das Recht erhalten habe, seinen Autor umzubringen. Das Schreiben, so lautet das etwas pathetische Argument des französischen Philosophen und Diskurstheoretikers, ist an das Opfer des Lebens gebunden, das in den Büchern nicht dargestellt werden soll, da es im Leben des Schriftstellers selbst sich vollzieht. Gemeint ist der Rückzug von Autoren wie Gustave Flaubert, Marcel Proust und Franz Kafka aus dem Leben um ihres Werks willen, der dazu führt, so Foucault, dass der Schriftsteller als Abwesender im Schreib-Spiel die Rolle des Toten zu übernehmen habe. ${ }^{8}$

5 Vgl. Martina Wagner-Egelhaaf, „Auf der Intensivstation. Oder: Die Autormaschine. Zu John von Düffels Missing Müller (Müllermaschine) (1977)“, in Martin Hellmold [u.a.] (Hgg.), Was ist ein Künstler? Das Subjekt der modernen Kunst, München 2003, 195-211, hier $207 \mathrm{f}$.

6 In diesem Sinn hat Norbert Eke das Stück als „Absage an eine zunehmend vom Verlust ihres Sinnzentrums bedrohte ästhetische Praxis sowie die Rolle des ,wissenden “ und vorplanenden Autors“ interpretiert. Vgl. Norbert Eke, Heiner Müller. Apokalypse und Utopie, Paderborn 1989, 100.

7 Wilhelm Genazino, Das Bild des Autors ist der Roman des Lesers, Münster 1994, 35. 
Der tote Autor stellt nach Foucault, anders als Roland Barthes den, Tod des Autors verstanden wissen wollte, die implizite Figur des zeichenhaft verfassten Texts dar. Will sagen: Der vorliegende manifeste Text ist gleichsam die Signatur des Autors, der sich um seines Textes willen aus dem Leben zurückgezogen hat und also ,gestorben ' ist, um seinem Text zum Leben zu verhelfen bzw. selbst in seinem Text als (gesellschaftlich) Toter weiterzuleben. Roland Barthes hatte in seinem Der Tod des Autors betitelten Essay aus dem Jahr 1968 mit der wirkungsvollen Metapher vom Tod des Autors, die rasch zum viel zitierten Schlagwort wurde, die Ablösung der Kategorie des ,Autors“ durch die des ,Textes' als Bezugsgröße für die Auseinandersetzung mit literarischen Texten beschrieben. Im Text kreuzen sich nach Barthes die Linien des kulturellen Archivs, dessen Agent der zum Schreiber degradierte Autor wird. In der Debatte über den ,Tod des Autors ' werden Barthes und Foucault meist in einem Atemzug genannt, die konzeptionellen Unterschiede zwischen ihren je verschiedenen Begründungen für das ,Sterben ' des Autors finden in der Regel keine Berücksichtigung. Im Anschluss an Foucault entwickelt Genazino in sieben Schritten eine Dialektik des Autoren-Fotos. Sie lauten folgendermaßen:

1. Der Autor opfert, indem er schreibt, langsam sein Leben hin.

2. Die Selbstopferung fließt als Thema nicht (oder nur marginal) in das Werk ein.

3. Das Werk wird damit zu einer Entschuldigung für die Entfernung des Autors von den anderen Menschen, für seine Abwesenheit.

4. Die Abwesenheit und der Drang, sich für sie entschuldigen zu wollen, nötigt den Autor zur Selbstdarstellung, zur Selbstabbildung.

5. Das immerzu sich entschuldigende Abbild übernimmt die Funktion der Stellvertretung.

6. Durch seine fortdauernde Wiederkehr wird das Bild mehr und mehr zum Beleg für das Verschwinden des Autors, obwohl

7. gerade durch das Bild der Schein der Anwesenheit simuliert wird. ${ }^{9}$

Dem Bildnis des Autors kommt also eine doppelte Funktion zu: Zum einen substituiert es den abwesenden, sich um seines Werkes willen aus dem Leben zurückziehenden Autor, zum anderen bezeichnet es gerade seine Abwesenheit. Oder nochmals anders gesagt: Die mediale Reproduktion des Autor-Bildes wird zum Beleg für die Abwesenheit des Autors, obwohl das Bild bzw. die vielen Bilder, die von einem Autor zirkulieren, den Schein der Anwesenheit simulieren. ${ }^{10}$ Die Begriffe und Kategorien, die in dieser Debatte zum Einsatz kommen - ,Opfer', ,Anwesenheit', ,Abwesenheit" - konnotieren auf bemerkenswerte Weise den Bedeutungsbereich des Religiösen.

Bereits die autorschaftskritischen Positionen von Roland Barthes und Michel Foucault operieren in hohem Maße mit religiösen Metaphern, deren diskursive Funktion in der

\footnotetext{
8 Michel Foucault, „Was ist ein Autor?“, in Fotis Jannidis [u.a.] (Hgg.), Texte zur Theorie der Autorschaft (RUB 18058), Stuttgart 2000, 198-229, hier 204; vgl. Wilhelm Genazino, Das Bild des Autors, $36 \mathrm{f}$.

9 Wilhelm Genazino, Das Bild des Autors, 37.

$10 \mathrm{Vgl}$. ebd.
} 
Forschung nicht hinreichend diskutiert wurde: Barthes’ Anliegen, das Bild des Autors zu entsakralisieren ${ }^{11}$, versteht sich dezidiert als gegentheologisches Projekt:

Heute wissen wir, dass ein Text nicht aus einer Reihe von Wörtern besteht, die einen einzigen, irgendwie $^{12}$ theologischen Sinn enthüllt (welcher die ,Botschaft‘ des Autor-Gottes wäre), sondern aus einem vieldimensionalen Raum, in dem sich verschiedene Schreibweisen [écritures], von denen keine einzige originell ist, vereinigen und bekämpfen. [...] Genau dadurch setzt die Literatur (man sollte von nun an besser sagen: die Schrift), die dem Text (und der Welt als Text) ein, Geheimnis', das heißt einen endgültigen Sinn, verweigert, eine Tätigkeit frei, die man gegentheologisch und wahrhaft revolutionär nennen könnte. Denn eine Fixierung des Sinns zu verweigern, heißt letztlich, Gott und seine Hypostasen (die Vernunft, die Wissenschaft, das Gesetz) abzuweisen. ${ }^{13}$

Der ikonoklastische Akt, den Barthes am Bild des Autors verübt, hat, indem er gegentheologisch und wahrhaft revolutionär sein will, religiöse und politische Dimensionen. ${ }^{14}$ Natürlich ist das ,Bild“ des Autors auch eine Metapher und da die Metapher auch ein Bild ist, erweist sich die Rede vom ,Bild des Autors` als rhetorische Kippfigur zwischen pictura und figura. Dies inszeniert paradigmatisch die eingangs zitierte Zerreißung der Fotografie des Autors ${ }^{15}$ : Ein materiales Bild, eine Fotografie (pictura), wird zerrissen, gemeint ist aber auch die abstrakte Vorstellung oder Instanz des Autors, wie sie sich in den Text eingeschrieben hat (figura). In diesem Sinn spricht auch Goethe im Vorwort seiner Autobiographie Dichtung und Wahrheit vom Bild des Autors: Er zitiert einen fiktiven Brief von Freunden, die ihn angeblich gebeten haben, sein Leben aufzuschreiben:

Man kann sich nicht enthalten, diese zwölf Bände, welche in Einem Format vor uns stehen ${ }^{16}$, als ein Ganzes zu betrachten, und man möchte sich daraus gern ein Bild des Autors und seines Talents entwerfen. Nun ist nicht zu leugnen, daß für die Lebhaftigkeit, womit derselbe seine schriftstelleri-

11 Roland Barthes, ,Der Tod des Autors“, in Fotis Jannidis [u. a.] (Hgg.), Texte zur Theorie der Autorschaft (RUB 18058), Stuttgart 2000, 185-193, hier 188.

12 Das ,Irgendwie“ des Vergleichs mit dem religiösen Bereich nutzt religiöse Vorstellungen und Bilder ohne eine Identifizierung vorzunehmen, stellt aber das europäische Autorschaftsdenken historisch und systematisch in einen religiös-theologischen Diskurszusammenhang.

13 Roland Barthes, „Der Tod des Autors“, 190f.

14 Dass die poststrukturalistische Verabschiedung des Autor-Subjekts politische und religiöse Dimensionen verbindet, zeigt etwa auch Martin Mosebachs Bezeichnung des Jahres 1968 als „Achsenjahr“: „Studentenrevolten in Deutschland, Frankreich, in den Vereinigten Staaten; der Beginn der chinesischen Kulturrevolution mit Millionen Toten, mit ihrer Bilderstürmerei, der Verwüstung von Tempeln und Kunstschätzen - und das Jahr der Liturgiereform“, Martin Mosebach, „,, Die Bilder aus den Herzen reißen“. Bildersturm und Liturgie“, in Ders., Häresie der Formlosigkeit. Die römische Liturgie und ihr Feind, München 2007, 70-88, hier 76. Vgl. auch Ulrich Horstmann, Ausgewiesene Experten. Kunstfeindschaft in der Literaturtheorie des 20. Jahrhunderts, Frankfurt a.M. 2003, 25f.: „Barthes' Liquidationsanzeige erschien 1968, im Jahr der Revolte und des Aufbegehrens, und wie die Studenten gegen die politischen Institutionen Sturm liefen, so unternahm es dieser antiautoritäre Theoretiker, das Ancien régime der Literatur vom Sockel zu stoßen und den ,Tod des Autors' als fait accompli erscheinen zu lassen.“

15 Heiner Müller, „Hamletmaschine“, 96.

16 Gemeint ist die bei Cotta in Tübingen 1806-1808 erschienene zwölfbändige Ausgabe von Goethes Werken. 
sche Laufbahn begonnen, für die lange Zeit die seit dem verflossen, ein Dutzend Bändchen zu wenig scheinen müssen. Eben so kann man sich bei den einzelnen Arbeiten nicht verhehlen, daß meistens besondere Veranlassungen dieselben hervorgebracht, und sowohl äußere bestimmte Gegenstände als innere entschiedene Bildungsstufen daraus hervorscheinen, nicht minder auch gewisse temporäre moralische und ästhetische Maximen und Überzeugungen darin obwalten. Im Ganzen aber bleiben diese Produktionen immer unzusammenhängend; ja oft sollte man kaum glauben, daß sie von demselben Schriftsteller entsprungen seien. ${ }^{17}$

Die Freunde möchten also aus dem Werk ein Bild des Autors gewinnen. Das Werk aber ist unzusammenhängend, deshalb bedarf es eines autobiographischen Texts, der ein ,Bild“ des Autors im übertragenen, im figurativen Sinne zeichnet. Und über dieses Bild wird nun wiederum das Werk als Einheit verständlich. Das ist gute hermeneutische Tradition. Foucault hätte sich dadurch bestätigt gesehen, weist er doch darauf hin, dass eine wesentliche Rolle der Autorfunktion darin besteht, die Einheit eines Werks zu begründen. ${ }^{18}$ Foucault hat Barthes bekanntlich kritisiert, indem er die von letzterem vorgenommene Ersetzung der Kategorie ,Autor' durch diejenige des ,Schreibers' als transzendentale[] Blockierung ${ }^{19}$ bezeichnete. Er führt aus:

Wenn man nämlich dem Schreiben ein ursprüngliches Statut zuweist, so ist das wohl nur eine Art, einerseits die theologische Behauptung vom geheiligten Charakter des Geschriebenen und andererseits die kritische Behauptung seines schöpferischen Charakters ins Transzendentale rückzuübersetzen. [...] wenn man das Schreiben als Abwesenheit begreift, heißt das dann nicht einfach, in transzendentalen Worten das religiöse Prinzip der zugleich unwandelbaren und nie erfuillten Tradition und das ästhetische Prinzip vom Überleben des Werks, von seinem Fortbestand über den Tod hinaus, von seinem rätselhaften Überschuß im Verhältnis zum Autor wiederholen ${ }^{20}$

An einer späteren Stelle seiner programmatischen Schrift kommt Foucault - und der Zusammenhang liegt nahe - auf das Urheberrecht zu sprechen. Er behauptet, dass die Rede am Ursprung unserer Kultur [...] kein Produkt, keine Sache, kein Gut war; sondern wesentlich [...] ein Akt, der seinen Platz hatte in der Bipolarität des Heiligen und Profanen, des Erlaubten und Verbotenen, des Religiösen und Blasphemischen. ${ }^{21}$ Seitdem der Autor Ende des 18., Anfang des 19. Jahrhunderts im Zuge der Ausformulierung des Urheberrechts zum Eigentümer seiner Texte wurde, scheint er, so stellt Foucault fest, den erreichten Status durch die Rückkehr zur alten Bipolarität der Rede, durch systemati-

17 Johann Wolfgang von Goethe, Aus meinem Leben. Dichtung und Wahrheit, hg. v. Klaus-Detlef Müller, Sämtliche Werke in 40 Bdn., Bd. 1/14, Frankfurt a.M. 1986, 11.

18 Michel Foucault, „Was ist ein Autor?“, 217f.: Der Autor ist [...] das Prinzip einer gewissen Einheit des Schreibens, da alle Unterschiede mindestens durch Entwicklung, Reifung oder Einfluß reduziert werden. Mit Hilfe des Autors kann man auch Widersprüche lösen, die sich in einer Reihe von Texten finden mögen: es muß da-in einer gewissen Schicht seines Denkens oder seines Wünschens, seines Bewußtseins oder seines Unterbewußtseins - einen Punkt geben, von dem her sich die Widersprüche lösen, an dem sich die unvereinbaren Elemente endlich verketten lassen oder sich um einen tiefen und ursprünglichen Widerspruch gruppieren.

19 Ebd. 207.

20 Ebd. 206f.

21 Ebd. 211. 
sche Übertretung, durch die Wiederherstellung der Gefahr beim Schreiben kompensieren zu wollen. ${ }^{22}$ Autorschaft ist also nicht einfach ungebrochene Fortsetzung eines in der Polarität von Heiligkeit und Profanität verorteten Redeakts, sondern Versuch der Rückgewinnung einer durch die Sicherung im Profanen, also im marktwirtschaftlich und juristisch gedachten Recht des Eigentümers, verloren gegangenen anderen Dimension, des Heiligen. Unter Bezugnahme auf die Authentizitätskriterien des heiligen Hieronymus verweist Foucault darauf, dass die moderne Literaturkritik in ihrem Umgang mit dem Autor recht geradlinig abgeleitet [sei] von der Art, wie die christliche Tradition Texte beglaubigte. ${ }^{23}$

Es handelt sich um die folgenden Kriterien, die Foucault der Schrift De viris illustribus des heiligen Hieronymus entnimmt, der mithin als ein erster Autorschaftstheoretiker in Anspruch genommen wird. Der Autor muss erstens ein bestimmtes konstantes Wertniveau darstellen, zweitens als Feld eines begrifflichen und theoretischen Zusammenhangs verstanden werden können, drittens als stilistische Einheit erscheinen und viertens als ein bestimmter geschichtlicher Augenblick und Schnittpunkt einer Reihe von Ereignissen wahrnehmbar werden. ${ }^{24}$

22 Ebd. 212. Auch Giorgio Agamben verbindet in seinem Essay „Der Autor als Geste“ (durchaus im Anschluss an Foucault) Autorschaft mit der Kategorie des Risikos, wenn er schreibt: Der Autor markiert den Punkt, wo sich ein Leben im Werk aufs Spiel gesetzt hat. Aufs Spiel gesetzt, nicht ausgedrückt; aufs Spiel gesetzt, nicht erfüllt. Deshalb kann der Autor im Werk nur unbefriedigt und unerwähnt bleiben. Er ist der Unlesbare, der das Lesen ermöglicht, die legendäre Leere, von der das Schreiben und der Diskurs ausgehen. Die Geste des Autors wird im Werk, das er trotz allem ins Leben ruft, als eine unangemessene, fremde Anwesenheit bestätigt, genauso wie nach der Ansicht der Theoretiker der commedia dell'arte Harlekins Scherze unaufhörlich die Geschichte unterbrechen, die auf der Bühne vor sich geht, und hartnäckig deren Handlung zersetzen. Doch genau wie nach der Ansicht derselben Theoretiker der Scherz, auf italienisch lazzo (die Schlinge), seinen Namen der Tatsache verdankt, daß er, wie eine Schlinge, den Faden, den er aufgebunden und gelockert hat, immer wieder anknüpft, garantiert die Geste des Autors das Leben des Werks allein durch die irreduzible Anwesenheit eines ausdruckslosen Randes. Wie der Mime in seinem stummen Spiel, wie Harlekin mit seinem lazzo schließt er sich unermüdlich immer wieder in das Offene ein, das er selbst geschaffen hat. Und wie in manchen alten Büchern neben dem Frontispiz ein Porträt oder eine Fotografie des Autors gezeigt wird und wir in seinen rätselhaften Zügen vergeblich die Gründe und den Sinn des Werks zu entziffern versuchen, so zögert die Geste des Autors auf der Schwelle zum Werk als ein unzugängliches ex ergon, das ironisch darauf pocht, dessen uneingestehbares Geheimnis zu besitzen. Giorgio Agamben, „Der Autor als Geste“, in Ders., Profanierungen, Frankfurt a.M. 2005, 57-69, hier 66.

23 Vgl. Michel Foucault, ,Was ist ein Autor?“, 215; vgl. Michelangelo Caravaggio (1571-1610), Der heilige Hieronymus beim Schreiben, http://de.wikipedia.org/wiki/Michelangelo_Merisi_da_Caravaggio, (letzter Aufruf am 30.05.2011).

24 Vgl. Hieronymus und Gennadius, De Viris inlustribus, hg. v. Lic. Carl Albrecht Bernoulli, Freiburg i.B./Leipzig 1895. Foucault extrahiert die genannten Kriterien aus Ausführungen wie der folgenden aus dem 15. Kapitel von Hieronymus' Werk, wo es über den Bischof Clemens heißt: Scripsit ex persona Romanae ecclesiae ad ecclesiam Corinthiorum ualde utilem epistulam et quae in nonnullis locis etiam publice legitur, quae mihi uidetur characteri epistulae, quae sub Pauli nomine ad Hebreos fertur, conuenire; sed et multis de eadem epistula non solum sensibus, sed iuxta uerborum quoque ordinem abutitur; et omnino grandis in utraque similitudo est. Fertur et secunda ex eius nomine epi- 


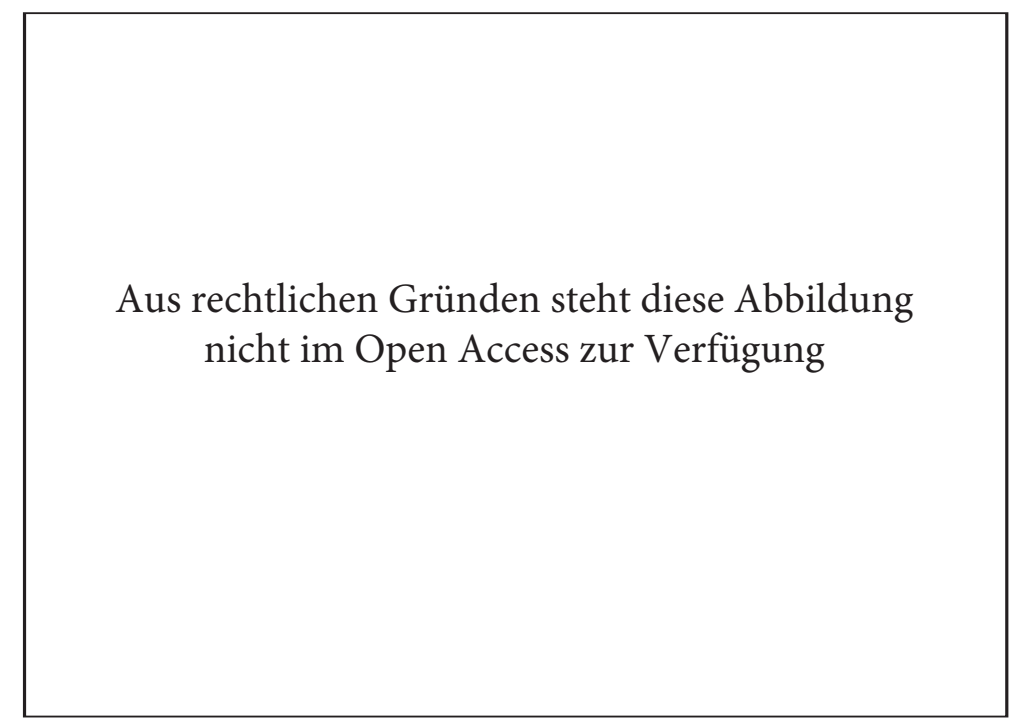

Abb. 1: Michelangelo Caravaggio (1571-1610), Der heilige Hieronymus beim Schreiben, 1605/06, Galleria Borghese, Rom

Schließlich verweist Foucault, dessen Essay üblicherweise viel zu thesenartig und verkürzt rezipiert wird, auf die Paradoxie, dass einerseits im Autor als dem Schöpfer eines Werks, der so anders ist als alle anderen Menschen und so transzendent bezüglich aller Sprachen $^{25}$ eine Quelle unendlicher Bedeutungsvermehrung gesehen und er doch nach den Maßgaben der Authentizitätskontrolle als bedeutungslimitierendes Prinzip eingesetzt wird. Die Funktion des Diskurses steht hier im eklatanten Widerspruch zu seiner offiziell vorgetragenen Ideologie. Als Funktion hat der Autor nichts mit dem wirklichen Schriftsteller oder dem fiktionalen Sprecher eines Textes zu tun, ist vielmehr gerade in diesem Bruch - in dieser Trennung und Distanz ${ }^{26}$ zu suchen. Als Funktion ist er die Figur einer Verknappung, die gegen das nach Barthes zu entsakralisierende ideologische Bild des Autors als transzendenter, Bedeutungsvermehrer'steht.

Die formalistische Literaturtheorie hat bekanntlich wesentliche Einsichten der poststrukturalistischen Autorschaftskritik vorweggenommen. Boris Tomaševskij etwa hat darauf hingewiesen, dass sich in der Romantik Name und Person des Autors nach vorn schieben und sich das Interesse des Lesers [...] vom Werk auf den Schöpfer ${ }^{27}$ ausdehnt. Als Folge wird das vom Autor geführte Leben literarisiert, Dichter werden zu Figuren vor

stula, quae a ueteribus reprobatur, et disputatio Petri et Appionis longo sermone conscripta, quam Eusebius in tertio ecclesiasticae historiae uolumine coarguit. Ebd. 17.

25 Michel Foucault, „Was ist ein Autor?“, 228.

26 Ebd. 217.

27 Boris Tomaševskij, „Literatur und Biographie“, in Fotis Jannidis [u. a.] (Hgg.), Texte zur Theorie der Autorschaft (RUB 18058), Stuttgart 2000, 49-61. 
dem Publikum ${ }^{28}$, und literarische Figuren, so Tomaševskij, werden für lebende Personen gehalten. Mit ihrem Leben, schreibt er, verwirklichten die Dichter eine literarische Aufgabe ${ }^{29}$ Der kategoriale Unterschied zwischen dem Autor als dem historisch-personalen Urheber eines Werks und dem Autor als Figur wird damit aufgehoben: Der Autor wird im beschriebenen romantischen Paradigma selbst zum Werk und das literarische Werk erscheint als Spiegel des Autors. Dagegen hat bereits Jan Mukařovský geltend gemacht, dass ein literarischer Text zwar einen Urheber hat, zwischen Künstler und Werk aber kein direkter Zusammenhang besteht. ${ }^{30}$ Dennoch war es die metaphorische Rede vom , Tod des Autors', die der Autorkritik disziplinübergreifende Aufmerksamkeit und dazu ein Label verschafft hat, unter dem die Debatte im Archiv der Literaturtheoriegeschichte abgespeichert werden konnte. Das ist insofern bemerkenswert als die biographische Metapher vom ,Tod des Autors' das Bild vom realiter getöteten Autor vor die theoretische Position schiebt, ${ }^{31}$ die mit diversen und sehr differenzierten Argumenten die Autorschaftsinstanz relativiert hat. Und die Metapher ist so wirkmächtig, dass das erneute literaturtheoretische Interesse am Autor in den 1990er-Jahren unter dem Bild seiner, Wiederkehr', ja sogar seiner, Wiederauferstehung' diskutiert wurde. ${ }^{32}$

Die Rede vom ,Bild des Autors ' ruft u. a. die umstrittene Figur des ,impliziten Autors auf. Wayne C. Booth hat argumentiert (und Seymour Chatman ihm sekundiert), dass jeder Autor eine Art zweites Ich in seinen Text hineinlegt, das weder mit dem historischen Autor noch mit dem Erzähler identisch ist. Booth spricht von einem Bild, das das Werk vom Künstler vermittelt ${ }^{33}$ :

28 Ebd. 54.

29 Ebd. 55.

30 Jan Mukařovský, „Die Persönlichkeit in der Kunst“, in Fotis Jannidis [u.a.] (Hgg.), Texte zur Theorie der Autorschaft (RUB 18058), Stuttgart 2000, 65-79, hier 76.

31 Vgl. auch Martina Wagner-Egelhaaf, ,,, Dead Author's Identity in Doubt; Publishers Defiant ‘. Zu Uwe Johnsons Selbstnachruf“, in Rudolf Suntrup [u.a.] (Hgg.), Usbekisch-deutsche Studien III: Sprache - Literatur - Kultur - Didaktik, 4. usbekisch-deutsche Tagung Münster 23.-25. November 2009, Teilband 1: Begegnung von Orient und Okzident in der Literatur, Linguistik und Varietäten, Münster 2010, 201-213.

32 Vgl. Fotis Jannidis [u. a.], ,,Rede über den Autor an die Gebildeten unter seinen Verächtern. Historische Modelle und systematische Perspektiven“, in Dies. (Hgg.), Rückkehr des Autors. Zur Erneuerung eines umstrittenen Begriffs, Tübingen 1999, 3-35; Niels Werber, Ingo Stöckmann, „Das ist ein Autor! Eine polykontexturale Wiederauferstehung“", in Henk de Berg, Matthias Prangel (Hgg.), Systemtheorie und Hermeneutik, Tübingen/Basel 1997, 233-259.

33 Wayne C. Booth, „Der implizite Autor“, in Fotis Jannidis [u.a.] (Hgg.), Texte zur Theorie der Autorschaft (RUB 18058), Stuttgart 2000, 142-152, hier 146. Vgl. Seymour Chatman, Coming to Terms. The Rhetoric of Narrative in Fiction and Film, Ithaca/London 1990. Zu einer Kritik des ,impliziten Autors“vgl. Ansgar Nünning, ,Renaissance eines anthropomorphisierten Passepartouts oder Nachruf auf ein literaturkritisches Phantom? Überlegungen und Alternativen zum Konzept des ,implied author“ “, DVjs 67/1 (1993), 1-25. Zum ,implied author` vgl. auch den Beitrag von Bruno Quast in diesem Band, 133-144. 
[...] klar ist, daß das Bild, das sich der Leser von dieser Gegenwart macht, eine der wichtigsten Wirkungen des Autors ist. Wie unpersönlich er auch zu sein versucht, sein Leser wird sich unweigerlich ein Bild von dem offiziellen Schreiber konstruieren, der auf diese Art und Weise schreibt [...]. ${ }^{34}$

Es geht also um ein ,Bild“, das sich der Leser vom Autor macht und dessen Gegenwart er beim Lesen spürt. Dieses Bild ist nun kaum ein sehr konkretes (pictura), vielmehr wird es nach Booth über die in das Werk eingehenden moralischen Werte und Normen konstituiert, die sich dem Leser/der Leserin vermitteln, eine Art schattenhafter Figur (figura), die auch eine imaginäre Dimension hat und daher einen dritten Bildbegriff, denjenigen der imago, aufruft. Wenn der Leser im Akt des Lesens in einen imaginären Dialog mit dem impliziten Autor tritt, begibt er sich gleichsam in eine Spiegelbeziehung, in der sich der gelesene Text imaginär realisiert. ${ }^{35}$ Gérard Genette nimmt in seiner Kritik des Booth'schen Ansatzes die Rede vom ,Bild' auf, wenn er den implizierten Autor als das Bild $d[$ ies $]$ es Autors, wie es sich [...] auf der Basis des Textes konstruieren lie $\beta^{36}$, rekapituliert. Seine Frage lautet bekanntlich, ob der implizierte Autor eine notwendige Instanz zwischen dem Erzähler und dem realen Autor darstellt. Nach aller Logik, schreibt er, hat ein Bild nur dann andere Merkmale als sein Vorbild, verdient also nur dann eigens erwähnt zu werden, wenn es untreu, d.h. unrichtig ist. ${ }^{37}$ Was für Bilderstürmer aller Zeiten ein Argument gegen das Bild gewesen ist, nimmt Genette hier als Kriterium seiner Legitimität. Allerdings negiert er die Möglichkeit, dass ein Autor in seinem Text ein untreues Bild seiner selbst produzieren $^{38}$ kann und argumentiert, das vom (kompetenten) Leser konstruierte Bild sei treuer [...] als die Vorstellung, die der Autor sich von sich selbst machte. ${ }^{39}$ Daraus zieht Genette den folgenden Schluss: So gesehen also ist der implizierte Autor der authentische reale Autor. Schreiben wir, damit es wissenschaftlicher aussieht: IA = RA. Damit aber wird $I A$, das treue und folglich transparente Bild, zu einer überflüssigen Instanz. Exit IA. ${ }^{40} \mathrm{Ob}$ Genette mit seiner witzig-lakonischen Formulierung Exit IA Shakespeares Hamlet und das Abtreten von Hamlets Vater-Geist Exit Ghost zitiert, ${ }^{41}$ kann nicht nachgewiesen werden,

34 Wayne C. Booth, „Der implizite Autor“, 143.

35 An dieser Stelle soll nicht weitergehend mit Lacans Spiegelstadium-Modell argumentiert werden.

36 Gérard Genette, „Implizierter Autor, implizierter Leser?“, in Fotis Jannidis [u.a.] (Hgg.), Texte zur Theorie der Autorschaft (RUB 18058), Stuttgart 2000, 233-246, hier 235; vgl. 236.

37 Ebd. 237.

38 Ebd. 238.

39 Ebd. 239.

40 Ebd. Auch im Falle des ironischen Erzählens ist das Bild des Autors, das der Leser durch Erkennen der Ironie aus dem Text gewinnt, der reale Autor; nochmals also die Formel: ,IA = RA, und exit IA ', ebd. 241.

41 Den Hinweis verdanke ich Christian Sieg. Vgl. Shakespeare, Hamlet. Prinz von Dänemark, Englisch und Deutsch, in der Übersetzung von Schlegel und Tieck, hg. v. Levin L. Schücking, mit einem Essay ,Zum Verständnis des Werkes“ und einer Bibliographie von Wolfgang Clemen, Hamburg 1977, I/1, 51; I/1, 141; I/V, 92. Freilich treten bei Hamlet alle Figuren mit exit oder exeunt ab; gewiss ist aber das Auf- und Abtreten des Geistes besonders wirkungsvoll, insofern als der Geist aus einer anderen Realität kommt und wieder in sie abtritt. Das heißt das Erscheinen und Verschwinden des Geistes ist jedes Mal ein Akt, der die sog. ,Wirklichkeit' perforiert und in Frage stellt. 
in jedem Fall aber konzeptualisiert er den impliziten Autor als eine Bühnenfigur, die offensichtlich Auftritte und Abgänge im Text hat. Mit Shakespeare gelesen eignet der Figur des impliziten Autors unzweifelhaft etwas Gespensterhaftes, das nicht die Figuren im Text wie bei Hamlet, sondern eher die Leser/innen und Interpreten bzw. Interpretinnen des Texts heimsucht. Noch in Genettes weiterführender Argumentation findet das Gespenst des impliziten Autors seinen Widerhall: Untreue Bilder des Autors konstruiert der Leser nach Genette nämlich nur in drei Ausnahmefällen: dem Apokryph, der Ghostwriterschaft und der Doppelautorschaft - alles zweifelhafte, sich dem Prinzip fassbarer Eindeutigkeiten entziehende ,Erscheinungen'. Genette begründet seine generelle Ablehnung des impliziten Autors mit einem pragmatischen Argument. Er ist nämlich der Meinung, dass man die narrativen Instanzen nicht unnötigerweise vermehren solle. Der reale Autor und der Erzähler genügen - eine dritte Hypostase ist also nicht erforderlich. Anders denkt hier Umberto Eco, wenn es ihm darum geht, die Autonomie des Textes gegenüber seinem Urheber, dem empirischen Autor, zu begründen. Hinter dem, was er, Textintention ' nennt, steht aber auch ein Schatten-Autor, nämlich das Phantasma des exemplarischen Autors, der mit der Textintention identisch ist. Zwischen dem empirischen und dem exemplarischen Autor lässt er sogar den Gedanken an eine dritte, fast gespenstische Figur zu, einen Grenz- oder Schwellenautor, d.h. die Schwelle zwischen der Absicht eines leibhaftigen Menschen und der Sprachintention, die sich in einer Textstrategie niederschlägt. ${ }^{42}$ Ecos exemplarischer Autor ist eine Hilfskonstruktion, die es ermöglicht, den empirischen Autor aus dem Text hinauszukatapultieren und doch die Rationalität und Interpretierbarkeit des Textes zu sichern. Der exemplarische Autor ist reiner Geist: Intention und Strategie, der sich aber in der und als Sprache verkörpert hat.

Diese literaturtheoretischen Hypostasen des Autors im Text sind von einer hybriden, zwischen pictura, imago und figura schwankenden Bildlichkeit. Als Vorstellungsbilder ${ }^{43}$ zehren sie, wie Vampire, vom Bild des empirischen Autors als dem Schöpfer des Textes, den sie, um im Bild zu bleiben, zum Zwecke seiner theoretischen Delegitimierung ,aussaugen'. Meine Gedanken saugen den Bildern das Blut aus, konstatiert Heiner Müllers Hamletdarsteller entsprechend. ${ }^{44}$ Versucht man nun, die Metaphorik des ,Bilderstreits ' für die Theoriedebatte um die Autorschaft produktiv zu machen, muss freilich historisch und systematisch differenziert werden. ${ }^{45}$ Hans Belting hat das Kultbild im antagonistischen Feld zwischen Glaubenspraxis und theologischer Doktrin beschrieben.

Die Theologen haben immer wieder versucht, materiellen Bildern ihre Macht zu entreißen, wenn diese im Begriff waren, zuviel Macht in der Kirche zu gewinnen. Bilder waren unerwünscht, sobald sie größeren Zulauf erhielten als die Institutionen selbst und ihrerseits im Namen Gottes zu agieren

42 Umberto Eco, „Zwischen Autor und Text“, in Fotis Jannidis [u.a.] (Hgg.), Texte zur Theorie der Autorschaft (RUB 18058), Stuttgart 2000, 279-294, hier 281.

43 Genette spricht davon, dass Vorstellung [idée] [...] ein besserer Ausdruck als ,Bild ‘ [...] sei, Gérard Genette, ,Implizierter Autor, implizierter Leser?““, 244.

44 Heiner Müller, „Hamletmaschine“, 93.

45 Vgl. grundlegend Wolfgang Brückner, „Bild, Bilderverehrung, Bilderverbot, Bilderstreit“, in Walter Kasper (Hg,), Lexikon für Theologie und Kirche, Bd. 2, Freiburg ${ }^{3} 2006,440-449$. 
begannen. Ihre Kontrolle war mit verbalen Mitteln ungewiß, weil sie wie die Heiligen tiefere Schichten berührten und andere Wünsche erfüllten, als es die lebenden Kirchenmänner konnten. Deshalb lieferten die Theologen in Bilderfragen immer nur die Theorie einer schon bestehenden Praxis nach. Niemals führten sie die Bilder aus freien Stücken ein, aber sie verboten sie umso lieber. Nur wenn andere sie verboten hatten und damit gescheitert waren, führten sie die Bilder wieder ein, weil sie in den Wünschen der Gläubigen präsent geblieben waren. Ihre Zulassung konnte man dann an Bedingungen knüpfen, die garantierten, daß man den Überblick behielt. Wenn sie die Bilder ,erklärt" und den Zugang zu ihnen reguliert hatten, waren die Theologen zuversichtlich, die Dinge wieder in der Hand zu haben. ${ }^{46}$

Ersetzt man in dieser Darstellung ,Theologen` durch ,Literaturwissenschaftler', ,Bilder' durch ,Autoren“, ,Gott' durch ,Literatur' und ,Gläubige ‘ durch ,Leser/innen ‘, erhält man eine Beschreibung der literaturwissenschaftlichen Autorschaftsdebatte in den letzten fünfzig Jahren, die zumindest nicht falsch ist. Im kultischen Bild der Ära des Bildes, die Belting vor der Ära der Kunst ansetzt, tritt, so formuliert er, ,jemand in Erscheinung “47; und: man muss es, das Bild, nicht nur ansehen, sondern auch glauben. ${ }^{48}$ Wichtig ist der Anspruch des authentischen Aussehens, den die Ikone immer erhoben hat: Man wollte im Bildnis den Eindruck der Person gewinnen und suchte das Erlebnis der persönlichen Begegnung. Ikonen werden daher grundsätzlich nach, authentischen', historischen Vorbildern und nicht nach der Phantasie gemalt. ${ }^{49}$ Im byzantinischen Bilderstreit wurde die Zulässigkeit von Ikonen u. a. von Johannes von Damaskus mit der Inkarnation begründet. Gottes Menschwerdung in Christus ermöglichte das zuvor nicht erlaubte Gottesbild; insofern verletzt die Ikone nicht das in Ex 20,4f. formulierte Bilderverbot, ${ }^{50}$ sondern bestätigt es. Die orthodoxe Kirche hat lange Zeit konsequenterweise nur die bildliche Darstellung der Zweiten Hypostase (Gott als Sohn) zugelassen. ${ }^{51}$ Die Gegner des Bildes argumentierten also mit der Nichtdarstellbarkeit Gottes, während seine Befürworter in der Abbildung der menschlichen Natur Gottes die vermittelnde Leistung des Bildes stark machen konnten. Ikonoklastische Akte sind häufig politisch motiviert, etwa wenn das machtpolitische

46 Hans Belting, Bild und Kult. Eine Geschichte des Bildes vor dem Zeitalter der Kunst, München ${ }^{2} 1993$, 11.

47 Ebd. 19.

48 Ebd. 23.

49 Vgl. ebd.; vgl. Karl Christian Felmy, „Ikone/Ikonenmalerei“, in Hans Dieter Betz [u.a.] (Hgg.), Religion in Geschichte und Gegenwart. Handwörterbuch für Theologie und Religionswissenschaft, Bd. 4: I-K, Tübingen ${ }^{4} 2001,36-41$, hier 36, 38.

$50 \mathrm{Du}$ sollst dir kein Bildnis noch irgendein Gleichnis machen, weder von dem, was oben im Himmel, noch von dem, was unten auf Erden, noch von dem, was im Wasser unter der Erde ist, Die Bibel oder die ganze Heilige Schrift des Alten und Neuen Testaments nach der Übersetzung Martin Luthers, Stuttgart 1970, 83 (2. Mose 20,4).

51 Vgl. Felmy „Ikone/Ikonenmalerei“, 37. Der Autor Martin Mosebach behauptet, Gott selbst habe das Zweite Gebot des Dekalogs „Du sollst dir kein Bild machen“ in Jesus Christus aufgehoben: Du sollst dir von Gott ein Bild machen, und diese [sic] Bild ist Jesus Christus, Martin Mosebach, ,,, Die Bilder aus den Herzen reißen“ “, 82. Zum Bildbegriff des Johannes von Damaskus vgl. Christian Hecht, ,DDas Christusbild am Bronzetor. Zum byzantinischen Bilderstreit und zum theologischen Bildbegriff“, in Karl Möseneder (Hg.), Streit um Bilder. Von Byzanz bis Duchamp, Berlin 1997, 1-25, hier $11 \mathrm{f}$. 
Gleichgewicht zwischen Christusbild und Kaiserbild zu kippen drohte. Zu erinnern ist in diesem Zusammenhang an die Entfernung des Christusbildes vom Bronzetor des Kaiserpalastes in Konstantinopel durch Leo III. im Jahr 726, die den Beginn der ersten, kaiserlich verordneten ikonoklastischen Phase markiert. ${ }^{52}$ Doch ist das Bild auch in vormoderner Zeit textbezogen: „Die Bilder enthalten ein Moment der Erzählung, auch wenn sie keine Erzählung sind“", schreibt Belting. ,Das Bild ist [...] nur verständlich, wenn man es von der Schrift her wiedererkennt. Es erinnert daran, was die Schrift erzählt, und erlaubt zusätzlich den Kult der Person [...]. “53 In der Reformationszeit gewinnen, so führt Belting aus, die Theologen, die Prediger des Worts, die Oberherrschaft über das Bild. „Das Bild kam gegen den authentischen Wortlaut nicht an, sondern enthielt, wenn man es anstelle der Schrift benutzte, die Gefahr des falschen oder des undeutlichen Verständnisses. “54 Das Wort bildet nicht ab, sondern ist Zeichen der Verständigung. Die Reformation, die sich im Zeichen Zwinglis und Calvins (Luther nahm da eher eine vermittelnde Position ein) ikonoklastisch gegen Bilder wandte, leitet bereits über in die Ära der Kunst, die im Kultbild bestenfalls noch eine Metapher wahrnimmt, keinesfalls aber mehr die Unmittelbarkeit der Evidenz. ${ }^{55}$ Auch die Autorschaftsdebatte wird im Zeitalter der Kunst geführt. Die Bilder von Autorinnen und Autoren, die in der literarischen Öffentlichkeit der Gegenwart begegnen, und diesmal sind die materialen picturae gemeint, sind ihrerseits zu einem großen Teil durchaus als Kunstwerke zu betrachten, wiewohl ihr Einsatz auf dem literarischen Markt, in Verlagsprospekten und auf Buchcovern den Kunstcharakter der Autorenporträts funktionalisiert. Hier sollen die Autorenbilder helfen, Bücher zu autorisieren und zu verkaufen. Dabei präsentieren sie Personen, die nicht als Heilige verehrt, sondern als Künstlerinnen und Künstler geschätzt und bewundert werden. Der Kunstanspruch des modernen Bildes verkörpert sich gleichsam in der Figur des/r Abgebildeten. Sehen die Gläubigen im vormodernen Kultbild die dargestellte Figur, ist die moderne Wahrnehmung des Autorenporträts, das heute in der Regel ein fotografisches ist ${ }^{56}$, geprägt von einer doppelten

52 Vgl. Ulrike Brunotte, „Ikonoklasmus“, in Religion in Geschichte und Gegenwart, Bd. 4, 52 f.; Christian Hecht, „Das Christusbild am Bronzetor“, 2. Berthold Hinz beschäftigt sich mit der durch den Reichsdeputationshauptschluss von 1803 durchgesetzten Säkularisation der Kirchengüter und dem Übergang der religiösen Kunstwerke in den Besitz der bürgerlichen Gesellschaft. Bilderstürme, so argumentiert er, sind dort nicht notwendig, wo ein Gesellschaftsystem das andere ablöst. Die Rede vom ,Bildersturm ‘ bleibt in diesen Fällen metaphorisch. Vgl. Berthold Hinz, „Säkularisation als verwerteter ,Bildersturm‘. Zum Prozeß der Aneignung der Kunst durch die Bürgerliche Gesellschaft“, in Martin Warnke (Hg.), Bildersturm. Die Zerstörung des Kunstwerks, München 1973, 108-120, hier 119.

53 Hans Belting, Bild und Kult, 20. Im jüdischen Bilderverbot, das auf der Unsichtbarkeit Gottes insistiert, ist es die Heilige Schrift, die zur Ikone Gottes wird und zur kultbildgleichen Verehrung der Thorarolle führt (vgl. ebd. 17f.).

54 Ebd. 25.

55 Ebd. 26.

56 Vgl. dazu den sehr erhellenden Aufsatz von Matthias Bickenbach, „Fotografierte Autorschaft. Die entzogene Hand“, in Matthias Bickenbach/Annina Klappert/Hedwig Pompe (Hgg.), Manus loquens. Medium der Geste - Gesten der Medien, Köln 2003, 188-209. Vgl. auch die Habilitationsschrift von 
Perspektive, einem Oszillieren zwischen Darstellung (figura) und dargestellter Person (pictura). Die imago eines Autors/einer Autorin ,spukt' vermutlich noch dazwischen, ohne sich manifestieren zu können. Diese ambivalente Wahrnehmung der Fotografie hat Roland Barthes bekanntlich mit den Begriffen studium und punctum beschrieben. In der Perspektive des studium wird das Foto als Bildkomposition wahrgenommen, während das punctum jenes Moment ist, in dem ein Foto besticht und gleichsam die Oberfläche des Bildes durchbohrt. Nach Barthes ist das jener Augenblick, in dem der Betrachter oder die Betrachterin spürt, dass die abgebildete Person tatsächlich „,da“ gewesen ist, ${ }^{57}$ auch wenn und gerade weil sie im Moment der Bildbetrachtung abwesend ist. Die Evidenz des Fotos indiziert die Abwesenheit der abgebildeten Person, während umgekehrt das Foto als Bild-Zeichen die vergangene Präsenz des/r Fotografierten zu sehen gibt. Dass die vormoderne Bildwahrnehmung heute nicht gänzlich durch den kunstwissenschaftlichen Blick und die Autorität des Wortes ersetzt ist, macht freilich auch Belting geltend, wenn er davon spricht, dass der Mensch sich nie von der Macht der Bilder befreit habe. ${ }^{58}$

Was bedeutet das nun für den modernen Umgang mit dem Bild des Autors? Wie Belting festhält, dass das vormoderne Kultbild im Zeichen der Schrift gelesen wurde, argumentiert Genazino, um wieder auf ihn zu sprechen zu kommen, dass wer als Leser/in das fotografierte Gesicht eines Autors/einer Autorin betrachtet, glaubt, in diesen Gesichtern lesen zu können, was die Abgelichteten geschrieben haben. Genazino zufolge betrachten Leser/innen, die von bestimmten Texten berührt wurden, Bilder von Autorinnen und Autoren mit dem Verstehensziel: Wie ist der Autor zur besonderen Tiefe seiner Einsicht und damit meinem eigenen Selbstverstehen so nahe gekommen ${ }^{59}$ Zweifellos tritt hier die imago-Funktion des Autorbilds in Wirksamkeit. Genazino konstatiert aber auch, dass sich die laufende Veröffentlichung von Autorenporträts (picturae) als Werbestrategie vor die Texte schiebe und das Abbild als Kürzel für das Werk in der Medienwelt mit größerer Gewissheit überleben werde als das Werk selbst. ${ }^{60}$ Eine ähnliche Diagnose stellt Christian Schärf, für den „,die universale Überlagerung der Texte durch die Bilder“ längst eingetreten ist und der ,diese neue literarische Epoche das Zeitalter der Ikonostasen“ nennt,

Matthias Bickenbach, Das Autorenfoto in der Medienevolution. Anachronie einer Norm, München 2010.

57 Vgl. Roland Barthes, Die helle Kammer. Bemerkung zur Photographie, übers.v. Dietrich Leube, Frankfurt a.M. 1985, 35, 87.

58 Vgl. Hans Belting, Bild und Kult, 27; vgl. auch Wolfgang Brückner, „Bild, Bilderverehrung, Bilderverbot", 446.

59 Wilhelm Genazino, Das Bild des Autors, 11. Elke Heidenreich formuliert im Vorwort zu Isolde Ohlbaums Band Autoren Autoren. Ein Bilderbuch, Cadolzburg 2000, o. S.: „Ich kann in jedem dieser Gesichter, in jedem dieser Bilder endlos herumwandern und mir eine Geschichte dazu erfinden oder versuchen, das Rätsel zu lösen, das mir die Photos aufgeben.“

60 Vgl. Wilhelm Genazino, Das Bild des Autors, 27. Gertrud Fussenegger schreibt in Isolde Ohlbaums Band Fototermin. Gesichter der deutschen Literatur, Frankfurt a.M. 1984, 163: „Ich warne vor der Photographie. Sie überlagert die Bilder unseres eigenen Gedächtnisses, sie saugt sie auf. Am Ende erinnern wir uns nicht mehr an geliebte Menschen, sondern nur mehr an deren Bilder. Am Ende lagert unsere ganze Existenz in Albums, Kästen, Filmen.“ 
womit er „eine Literatur im vollendeten Zustand artifizieller Präsenz“ bezeichnet. ${ }^{61}$ Das lässt sich an den Bildbänden der Fotografin Isolde Ohlbaum veranschaulichen, die sich auf das Autorenporträt spezialisiert, ${ }^{62}$ aber auch Bildbände über Friedhofsskulpturen und Katzen veröffentlicht hat. Auf die Frage, worin das Spezifikum des Autorenporträts liegt, gibt die Autoren-Fotografin im Vorwort ihres Bandes Fototermin eine auf den ersten Blick überraschende Antwort:

Gibt es eine typische Physiognomie des Schriftstellers? Diese Frage wurde mir immer wieder gestellt. Sie hat mich beschäftigt, und ihre Beantwortung hatte - neben optischen, ästhetischen und formalen Kriterien - nicht geringen Einfluß auf die Auswahl der Fotos. [...] Die Vielfalt der Gesichter wollte ich zeigen $[. ..]{ }^{63}$

Das heißt es gibt eigentlich kein Spezifikum des Autorenporträts. Autorinnen und Autoren sind Individuen, deren Vielfalt die Fotografin offensichtlich reizt. Lakonisch stellt daher die Autorin Elfriede Czurda in dem genannten Band fest: Auch Schriftsteller schauen auf Fotos nur aus wie Menschen oder Unmenschen. Unverkennbar sehen sie jedoch aus wie Angehörige der herrschenden Kultur: mehr oder weniger gelungene Prototypen von Angestellten ${ }^{64}$ Tatsächlich stellt sich beim Betrachten der Porträts die Frage, ob die Abgelichteten nicht ganz normale Menschen sein könnten, Nicht-Autoren, Politiker, Wissenschaftlerinnen, Medienstars? Die folgenden, nahezu beliebig ausgewählten Beispiele aus Ohlbaums Fotoband Bilder des literarischen Lebens (2008) zeigen die Autorinnen und Autoren gerade nicht in Autorpose, mit einem Buch oder am PC. Bestenfalls signalisieren Grass' Pfeife oder die Zigarette in Herta Müllers Hand kritische Intellektualität.

Als Leserinnen und Leser betrachten wir diese Fotos mit einem gewissen voyeuristischen Interesse, insofern als sie uns mit sehr konkreten Menschen konfrontieren, die unser vages textgespeistes Autorbild auf irgendwie unpassende Weise supplementieren, wenn nicht überrumpeln. Die künstlich-künstlerische Präsenz berührt auf etwas peinliche Art und Weise, weil uns das Foto etwas vor Augen stellt, was wir eigentlich gar nicht sehen wollen und können. Die Person, die wir sehen, ist nämlich nicht ,der Autor ‘,die Autorin “ - bestenfalls sehen wir eine mit den Mitteln der fotografischen Kunst produzierte

61 Christian Schärf, „Belichtungszeit. Zum Verhältnis von dichterischer Imagologie und Fotografie“, in Gunter E. Grimm/Christian Schärf (Hgg.), Schriftsteller-Inszenierungen, Bielefeld 2008, 45-58, hier 57.

62 Vgl. Isolde Ohlbaum, Deutscher Literaturbetrieb heute. Bilder einer Branche, Berlin 1979; dies., Fototermin. Gesichter der deutschen Literatur, Frankfurt a.M. 1984; dies., Augen, Blicke. Augenblicke. Ein Photoalbum in Kupfertiefdruck, Nördlingen 1987; dies., Portraits. München 1993; dies., Im Garten der Dichter. Der Petrarca-Preis, München 1997; dies., Autoren Autoren. Ein Bilderbuch, Vorwort v. Elke Heidenreich, Cadolzburg 2000; dies., Bilder des literarischen Lebens. 352 Portraitphotographien aus vier Jahrzehnten von A-Z, mit einem Essay von Cees Nooteboom, München 2008; dies., Auswärtsspiele. Autoren unterwegs, Göttingen 2009.

63 Isolde Ohlbaum, Fototermin, o. S.

64 Ebd. 100. Zur Autorisierung mittelalterlicher Texte durch Autorenporträts vgl. Ursula Peters, „Digitus argumentalis. Autorbilder als Signatur von Lehr-Auctoritas in der mittelalterlichen Liedüberlieferung“, in Matthias Bickenbach/Annina Klappert/Hedwig Pompe (Hgg.), Manus loquens. Medium der Geste - Gesten der Medien, Köln 2003, 31-65. 
Aus rechtlichen Gründen steht diese Abbildung nicht im Open Access zur Verfügung
Abb. 2: Isolde Ohlbaum, Günter Grass (Berlin, 1980), in Bilder des literarischen Lebens, 96

Aus rechtlichen Gründen steht diese Abbildung nicht im Open Access zur Verfügung

Abb. 4: Isolde Ohlbaum, Herta Müller (Berlin, 2003), in Bilder des literarischen Lebens, 235

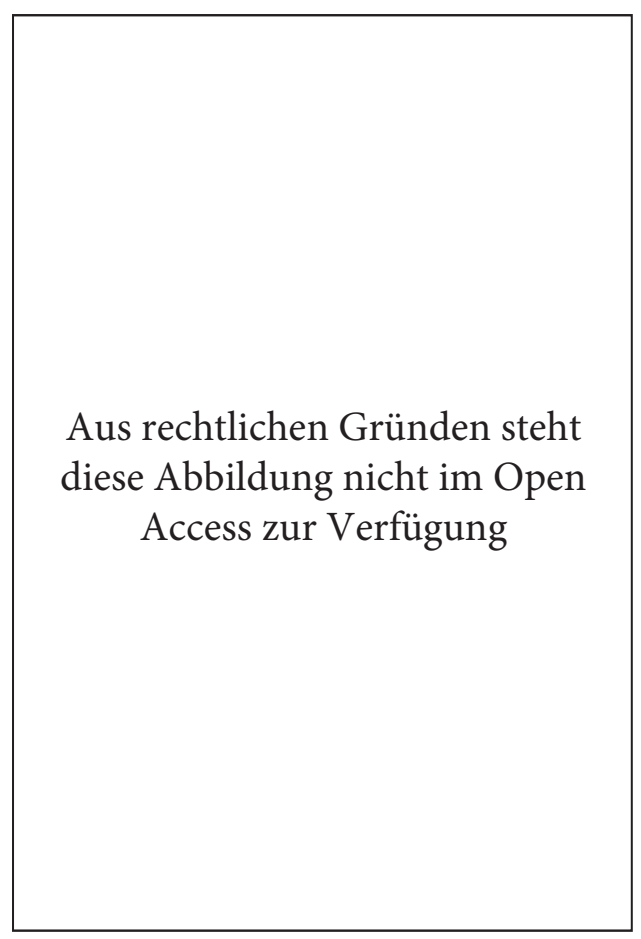

Abb. 3: Isolde Ohlbaum, Elfriede Jelinek (Wien, 1987), in Bilder des literarischen Lebens, 145

Aus rechtlichen Gründen steht diese Abbildung nicht im Open Access zur Verfügung

Abb. 5: Isolde Ohlbaum, Wilhelm Genazino (Darmstadt, 2004), in Bilder des literarischen Lebens, 87 
Version des/derselben. Zu Ohlbaums Foto-Band Bilder des literarischen Lebens hat Cees Nooteboom ein Vorwort geschrieben, in dem er hervorhebt, dass Ohlbaum die Autorinnen und Autoren gerade nicht zu gestellten Posen zwinge, sondern dass sie einen unfehlbaren Instinkt für die Gunst des Augenblicks habe, für die eine Sekunde, in der der Photographierende weiß, dass die Seele - es gibt kein besseres Wort für dieses ungreifbare Element dessen, der abgelichtet werden soll, optimal sichtbar wird. ${ }^{65}$ Dies lässt zweifellos an das metaphysisch-unverfügbare punctum-Moment bei Roland Barthes denken. Evoziert bereits der Bezug auf den fotografischen Moment der Seele den Bedeutungsbereich der Religion, rückt Ohlbaums Galerie der Abgelichtet-Verewigten einmal mehr in eine die Betrachter/innen des Bildbands einschließende Aura des Religiösen, wenn Nooteboom abschließend schreibt:

Die katholische Kirche kennt einen Ausdruck, der mich immer sehr angesprochen hat: die Gemeinschaft der Lebenden und der Toten. In diesem Buch hat Isolde Ohlbaum die Lebenden und die Toten versammelt, die sie im Laufe von vierzig Jahren photographiert hat. Eine Gemeinschaft bilden sie deshalb, weil ihnen allen, was auch immer sie voneinander halten mögen und wie unterschiedlich sie auch sein mögen, auf jeden Fall gemeinsam ist, daß sie sich von Zeit zu Zeit von der Welt abwenden, um in Gedichten, Romanen, Essays, Briefen und Tagebüchern von ebendieser Welt und von der Zeit ihres Lebens zu berichten. Daß sie letzteres mit jener gemeinsam haben, die sie - mit Licht schreibend-abgebildet hat, darin besteht das Wunder dieses Buches. ${ }^{66}$

Mit diesem Bild des von Zeit zu Zeit abwesenden Autors schließt Nooteboom wiederum an Genazinos Argument von der Substitution der auktorialen Anwesenheit an. Zweifellos eignen dem modernen Autorenbild Züge der alten Ikone, wenngleich im Zeitalter der Kunst die ins Zentrum der Seele treffende Identifikation immer kontrapunktiert wird durch die Wahrnehmung einer künstlerischen Leistung, des Bildes als Artefakt. Die Hypostasen des Autors, die die moderne Literaturtheorie geschaffen hat - der implizite Autor, der exemplarische Autor, der abstrakte Autor ${ }^{67}$, das vom Leser imaginierte ,Bild des Autors 'konstituieren ein geisterhaftes Zwischenwesen zwischen studium und punctum, pictura, imago und figura. Vielleicht ist ,der Autor' eben die Schwelle, wo die eine Perspektive in die andere umbricht. Streng genommen gibt es hier keinen Übergang, kein Dazwischen.

65 Cees Nooteboom, „Isolde Ohlbaum“, in Isolde Ohlbaum, Bilder des literarischen Lebens. 352 Portraitphotographien aus vier Jahrzehnten von $A-Z$, mit einem Essay von Cees Nooteboom, München 2008, 7-10, hier 9.

66 Ebd. 10. Auch Martin Mosebach bemüht in Bezug auf die von der Liturgiereform zerstörten Kirchenbilder „,die gestaltenreiche Communio sanctorum“, Mosebach, „,Die Bilder aus den Herzen reißen““, 73. Zum „,sog. Bilderzauber“ schreibt Wolfgang Brückner, er beruhe „nicht auf substituierender Wirkmächtigkeit, sondern dien[e] zeichenhafter Kommunikationsverstärkung“, Brückner, „Bilder, Bilderverehrung, Bilderverbot, Bilderstreit“, 448. Isolde Ohlbaum, die freundlicherweise den Abdruck ihrer Fotos in diesem Beitrag gestattet, korrigiert Nooteboom in einer mail vom 8.12.2010 dahingehend, dass es sich um Photos handelt, die innerhalb von dreißig Jahren, nämlich zwischen 1978 und 2008, entstanden sind.

67 Vgl. Cordula Kahrmann/Gunter Reiß/Manfred Schluchter, Erzähltextanalyse. Eine Einführung in Grundlagen und Verfahren, 2 Bde., Kronberg 1977, Bd. 1, 42. Jaap Lintvelt, Essai de typologie narrative. Le ,point de vue', Paris 1981, 17. 


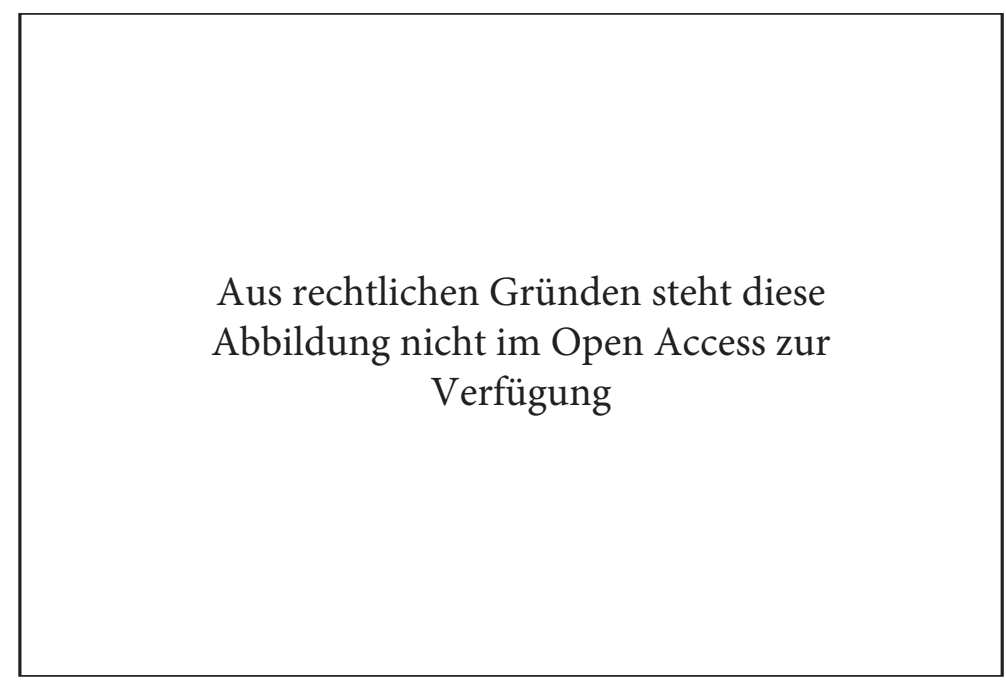

Abb. 6: Isolde Ohlbaum, Heiner Müller (Frankfurt, 1990), in Bilder des literarischen Lebens, 234

Es bedarf der pausenlosen metaphorischen Substitution, die aus der Person ein Bild, dem Bild einen Autor, dem Autor ein Werk, dem Werk einen Mörder und aus dem Getöteten einen Wiedergänger macht. Und um nochmals auf die eingangs erwähnte Zerreißung des Bilds des Autors in Heiner Müllers Hamletmaschine zurückzukommen, mag man es immerhin als tröstlich empfinden, dass die politischen Ikonoklasten, die Autorinnen und Autoren den Lebens- oder Schreibraum verweigern, deren ,wahres' Bild nicht erwischen können. 